\title{
RECOGNITION RULES IN WEIGHTED MAJORITY GAMES AND THEIR IMPLICATIONS
}

\author{
KRZYSZTOF KASPRZYK \\ Department of Mathematical Economy, Warsaw School of Economics \\ Al. Niepodlegtości 162, 02-554 Warszawa, Poland \\ E-mail: Krzysztof_Kasprzyk@mpips.gov.pl
}

\begin{abstract}
This paper examines implications of different random recognition rules used to select proposal-makers on the payoffs of players participating in a weighted majority game. In particular, incentives to strategically alter the set of players by strategic splits or mergers are investigated.
\end{abstract}

1. Introduction. The paper proposes a model which is a general case of the infinite bargaining problem analyzed by Rubinstein (1982) and is an extension to the model of Baron and Ferejohn (1989), in which players are selected randomly to make a proposal in a one-dimensional distributive space. Such proposals have to be approved by a winning coalition of voters.

Such a model can be used to describe both a legislative game in parliamentary democracies, in which a candidate for a prime minister (a formateur) is selected according to some recognition rule and needs approval from a majority of parliamentarians to install the proposed government, or a common-stock company in which the role of individual shareholders is analogous to the role of political parties in the parliamentary setting. Empirical evidence (see e.g. Diermeier and Merlo, 1999a) indicates that random recognition rules are appropriate for modeling such situations, which is contrary to the intuitive assumption that the order of selection of proposal-makers is affected through a rule that follows the ordering of weights of players, i.e. the biggest shareholder or political party is always selected first, with the rest following in a fixed order.

The paper studies different random recognition rules. Some normative and technical criteria used in the analysis are introduced. The rule that is the most popular in the literature is a proportional rule in which selection probabilities are proportional to weights assigned to each player. It is shown that it leads to non-zero expected payoffs for dummy

2000 Mathematics Subject Classification: 91A20, 91B12.

The paper is in final form and no version of it will be published elsewhere. 
players and hence possible surplus coalitions. As a general rule, this proportional rule provides the players with wide-spread incentives to strategically split or merge with other players. Other recognition rules studied in the paper are based on decisive structures, e.g. use power indeces such as Shapley values as recognition probabilities. While such rules eliminate the counter-intuitive property of surplus coalitions and dummy players with positive payoffs, they still fail to prevent the rise of abundance of incentives for strategic splits or mergers.

It is shown that there always exists a unique class of recognition rules that can prevent strategic splits or mergers. The formula and properties of such a "stable rule" are established. This uniqueness, and hence also relatively unlikely occurrence of such a split-and-merger-proof recognition rule, indicates that - while convenient for analytical reasons - the common assumption of fixing the set of players while modeling such games has important consequences and may be inconsistent with the incentive structure of the underlying bargaining problem.

2. The model. The model is based on Baron-Ferejohn model of bargaining. However some extensions and modifications are necessary to evaluate the possibility of splits or mergers.

First of all, in order to evaluate strategic splits and mergers, we cannot treat parties or shareholders as individual players, as is common in literature. We must go down to the individual legislators or stocks. On the other hand, the recognition rule to be used will be defined over the partition of this set of players into bigger groups - a party structure. Moreover, we assume that some initial party structure exists and each player is assigned to a single party. More formally:

Denote the set of players by $N=\{1,2, \ldots, n\}$. Those players are partitioned into $k$ parties, $1 \leq k \leq n$, i.e. each player belongs to one and only one party. A partition $K$ of the set $N$ will be called a party structure. For every game there exists an initial party structure denoted $K^{*}$. Weights of parties correspond to sizes of respective parties, i.e. numbers of players belonging to them. The elements of the set of players $N$ will be denoted by subscripts $i, j$ while elements of the set of parties $K$ will be denoted by subscripts $p, q$.

We will also define payoffs for individual players in their relation to the payoffs of their own parties. We will denote the payoff for a party $p$ under partition $K$ by $V_{p}$, $p \in K$, and the payoffs for individual players by $v_{i}, i \in N$. The payoff for a party is a sum of payoffs for all players belonging to the party, i.e. $V_{p}=\sum_{i \in p} v_{i}$. A sharing rule for a party $p$ will be a function $s$ which for every $V_{p}$ assigns a vector of payoffs to individual players belonging to that party such that $V_{p} \geq \sum_{i \in p} v_{i}$.

It remains to be defined how the individuals decide about possible splits, i.e. split rules. In general, for each party we can define a set of decisive coalitions $D$ and if a set of players that constitutes a decisive coalition strictly prefers some split to a status quo for a given initial party structure, we would say such a structure is vulnerable to splits. The most permissive rule would allow each individual to be the decisive coalition and a split would occur if there exists a single player that prefers such split to status quo. 
The most restrictive rule would require unanimity. The more restrictive the rule, the less likely maintaining of stability is.

The game would consist of two phases: in the first, organizational phase, splits are allowed. At the end of this phase the structure is fixed and no more splits are allowed between various rounds during the next phase. The detailed description of assumptions made about this phase is presented later. The organizational phase is followed by the bargaining phase. The latter phase follows the standard Baron-Ferejohn model: $k$ parties compete to divide a unit of good. At each round a party is selected according to a recognition rule $r$ to be a proposer. The proposer party makes a proposal specifying a particular division of the unit of good $\left(x_{1}, \ldots, x_{k}\right), \sum_{p=1}^{k} x_{p} \leq 1$. Then, each party accepts or rejects the proposal. If parties constituting a majority ${ }^{1}$ accepted the proposal at round $t$, each gets utility $\delta^{t-1} x_{p}$, with $\delta$ being a common discount factor, such that $\delta \in[0,1]$. Otherwise, the next round starts, another player is selected, makes a proposal and so on.

Austen-Smith and Banks (1988) first applied this type of model to bargaining in legislature. This paper follows main features of the model first introduced by Baron and Ferejohn (1989). As in the models mentioned earlier, parties bargain over a fixed amount of good. However, unlike in the traditional Rubinstein and Austin-Smith and Banks models, proposers (in the legislative settings called formateurs) are chosen randomly in each round. In general, a recognition rule would be a probability distribution specifying probability of being recognized as the proposer for each round, for each party structure and each party.

This paper aims at finding the recognition rules that are stable, i.e. if applied, the initial party structure $K^{*}$ is not vulnerable to splits and mergers. If a recognition rule lacks such property, it would imply there are incentives for parties to split or merge. Such strategic splits and mergers to increase one's bargaining power would put the common assumption of a fixed set of players into question.

3. The assumptions. In order to make the described model more tractable, several simplifying assumptions have been made. The assumptions and the rationale for choosing them are presented below.

- For the organizational phase, only 1 round is allowed. It means that given an initial partition $K^{*}$, players evaluate possible splits by comparing the payoffs they expect to get under $K^{*}$ with payoffs that result from the hypothetical split $K^{\prime}$. They do not have to consider implications of the splits for another round of splits etc. Additionally, thanks to this assumption, in order to find a stable rule (i.e. such that for any $K^{*}$ no party has an incentive to split) we only have to consider unilateral deviations by the party concerned against the status quo of $K^{*}$. A more general setup of the phase would allow for a much more complicated incentive structure which would interfere with the general picture. The minimalist model with this assumption is sufficient to create enough incentives to make splits almost universal, hence adding additional level of complexity is not necessary.

\footnotetext{
${ }^{1}$ We would require simple majority of players for an approval of a proposed division, but the model can be easily extended to supermajorities or more general decisive structures.
} 
- Furthermore, we allow each party to split into 2 smaller parties only. The assumption is conservative in terms of vulnerability to splits, as we simply consider a rule stable even if there are incentives for splitting into 3 or more parties. As with other conservative assumptions, since we will prove that splits are likely even under this assumption, such an assumption is reasonable.

- The sharing rule according to which players divide the payoff of their own party provides for an equal division of the payoff, i.e. if individual $i$ belongs to party $p$ of size $s_{p}$ and the party gets in round $t$ payoff $V_{p}$ the payoff for individual is given by $v_{i}=\frac{V_{p}}{s_{p}}$. Again this is a simplifying and conservative assumption. If even under this restrictive assumption splits are likely, then allowing for more general setup would make the structure even more vulnerable to splits.

- Another conservative assumption applies to split rules. Suppose that under some recognition rule and a structure $K$, the expected payoff for a party $p$ of size $s_{p}$ is $V_{p}$, i.e. $V_{p} / s_{p}$ per player belonging to the party. We will say a party has incentives to split only if expected average payoff per player in all the new parties formed from the old party is greater than before split. For a split into 2 parties on the party level that translates into the condition that

$$
V_{p}<V_{p 1}+V_{p 2}
$$

where $V_{p 1}$ and $V_{p 2}$ are the expected payoffs for the newly formed parties.

- We assume, again conservatively, that for a split to succeed the players must be strictly better off.

- The most important assumptions are buried beneath the shape of utility functions and choice space in general. First of all, we have only one, distributive dimension and players have no ideological positions, as in the original model of Baron and Ferejohn (1989). Some form of spatial choice space was considered for example by Baron (1991, 1993), Banks and Duggan (2000, 2001), while the paper by Jackson and Moselle (2002) gives interesting insights into the case with both ideological and distributive dimensions. Similarly, we assume the amount of good to be divided is fixed (and normalized to 1). That in particular leads to the no-delay property of the equilibrium: the game in equilibrium always ends in the first round ${ }^{2}$. If we follow Merlo and Wilson $(1995,1998)$ or Diermeier and Merlo (1999b) and make the amount of good stochastic, a similar model can explain possible failures of government-formation process in the first round which is more consistent with empirical evidence.

- We also need some assumptions on recognition rules. Since in other papers such a rule was generally fixed as proportional rule, i.e. probability of recognition for a party was equal to respective weights (share of seats/stocks each party holds), it is necessary to develop some more general conditions. The proposed assumptions are all satisfied by proportional rule and are as follows:

(i) Recognition rule is time-invariant. Although it is quite conceivable that this may be violated (e.g. if a proposer party that fails to get its proposal approved may be recognized with smaller probability in the next round), for simplicity we assume that the

\footnotetext{
${ }^{2}$ For discussion and simple proof, see Baron and Ferejohn (1989), pp. 1193-4.
} 
rule is time-invariant. This will give the natural meaning and power to the equilibrium concept we will use - that of a stationary subgame-perfect equilibrium - and will allow us to use directly the results from Baron and Ferejohn (1989) about the existence and uniqueness of such equilibria.

(ii) Recognition rule is anonymous, i.e. probabilities of recognition depend on weights of the parties only and if we just switch labels it should not affect the results. Again, we gain tractability, though it might be viewed as important simplification in the light of the estimation results of Diermeier and Merlo (1999b) indicating a significant incumbency premium ${ }^{3}$.

(iii) Recognition rule is weakly increasing, i.e. for a given party system $K$, for any pair of parties $p$ and $q$ if $s_{p}$ and $s_{q}$ denote their respective weights and $p_{p}$ and $p_{q}$ recognition probabilities, then $s_{p}>s_{q}$ implies $p_{p} \geq p_{q}$.

4. Solution concept. Once we apply the assumptions, our simplified model looks as follows:

1. Nature chooses initial party structure $K^{*}$.

2. Each party $p \in K^{*}$ selects a particular split into disjoint parties $p 1$ and $p 2$, i.e. $p=p 1 \cup p 2$. If no split occurs, it is represented by $p 1$ or $p 2=\emptyset$. The result is new party structure $K^{\prime}$.

3. Let the round $t=1$ start.

4. Party $q_{t}$ is selected according to the recognition rule $r$.

5. Party $q_{t}$ makes a proposal $x_{t}=\left(x_{1}, \ldots, x_{k^{\prime}}\right), \sum_{p=1}^{k} x_{p} \leq 1$.

6. Each party votes for or against the proposal.

7. If more players vote for than against the proposal, it is accepted. Each player belonging to party $p$ gets $\frac{\delta^{t-1} x_{p}}{s_{p}}$, where $s_{p}$ is a number of players belonging to party $p$. Otherwise, increase $t$ by 1 and go to item 4 .

The stages described in 3-7 are the same as in the standard Baron-Ferejohn (1989) model that was amended by a single split stage at the beginning of the game. We will use the concept of a stationary subgame-perfect equilibrium.

General properties of the solution (stationary subgame-perfect equilibrium) for the bargaining stage of the model follow directly from their paper. The equilibrium condition for each party $p$ is given by:

$$
p_{p}\left(1-\min _{C \in D_{p}}\left(\sum_{l \in C} \delta V_{l}\right)\right)+q_{p} \delta V_{p}=V_{p},
$$

with $V_{p}$ being the party's expected payoff from the game (i.e. its continuation value), $p_{p}$ is the party's recognition probability, $q_{p}$ is the probability of being asked by some other party to join a coalition, $\delta$ is a common discount factor and $D_{p}$ is given by

$$
D_{p}=\left\{C \in 2^{K^{\prime}}:|C \cup p|>n / 2\right\}
$$

i.e. in our case the set of all coalitions that together with party $p$ are decisive, since together they constitute a majority of $n$ players. It is easy to see that - if selected - each

\footnotetext{
${ }^{3}$ Diermeier and Merlo (1999b), p. 17.
} 
party distributes positive amounts only to the jointly cheapest coalition partners offering them $\delta V_{l}$ in order to make the parties indifferent between the offer and the parties' respective continuation values, hence indifferent between accepting and rejecting the proposal in that round. This selection corresponds to the values of $q_{p}$ in the equilibrium condition above. Solving the system of equations for each party we can find continuation values and probabilities $q$ for each party.

In the extended game which includes the additional organizational phase it is necessary to add just one additional condition for equilibrium: $K^{*}=K^{\prime}$. Notice that in order to find the equilibrium it is enough to consider each party's incentives to deviate from $K^{*}=K^{\prime}$ i.e. it is enough for each party to check possible splits for the party concerned assuming other parties do not split.

What follows is an examination of different recognition rules using the above model.

A. Proportional rule. According to this rule, the probability of recognition is equal to the weight, which is equal to the share of players belonging to each party. This rule is usually amended so that in the case a party has more that half of all players, the party is recognized with probability one. Such amended rule is the most common assumption made in literature. Note that in this case, and in many other cases considered later, even different probabilities of recognition might lead to the same continuation values. It is easy to understand what may go wrong with such rule if we consider the following very simple example:

EXAMPLE 1. There are initially 3 parties in the 30 -player set, each having the same number of 10 players. By the neutrality assumption, they all have the same continuation values of $1 / 3$. Suppose one of them considers a possible split into 2 parties of sizes 1 and 9 respectively. It is easy to check that in the new 4-party system all 3 parties having 9 or 10 seats have the same continuation values. The small party however, has non-zero continuation value, as in the event of being recognized it gets positive share of the unit of good. That implies the members of the splitting party are better off.

The benefit in this example comes from the fact that a small new party is created without any significant changes to decisive structure of the game. Although the small party is never asked to join a majority coalition, it is nevertheless recognized with positive probability and gets positive expected payoff at expense of other parties. Hence, "dummy" parties get positive expected payoff and a surplus coalition might happen.

B. Rule dependent on decisive structure. The above example suggested that part of the problem with stability is related to the possibility of formation of parties that are never pivotal in a majority coalition necessary to accept a proposal. Note that the common assumption already excludes such parties from being recognized if a party has majority of players. The natural way would be to extend the requirement to other partitions. Hence, a party would be recognized with positive probability only if it can be pivotal, i.e. there exists a majority coalition such that if the party is excluded the coalition no longer has the majority of players. One possible candidate is to use Shapley values of parties as the probabilities of recognition. They naturally employ the pivotalness requirement, including the common assumption about majority party. They sum up to 1 , so no normalization 
is necessary in order to use them as probability measures. They reflect the feature that minority coalition has value 0 (cannot provide anything to its members) and majority coalition can divide whole unit among themselves, hence the value of any majority party is 1 . However, we have to be very careful to make the distinction between power and the Shapley values in this model. Although Shapley values are called "power indices", if used as proposed in this model they only represent recognition probabilities. The "real" power is represented by continuation values, which are determined by the probabilities, but are quite distinct. As in the case of proportional rule, different probabilities of recognition may lead to the same continuation values. The Shapley values are used here as an example of a much more general class of rules that are dependent solely on the decisive structure of the game.

As we can see from the next example, making recognition probabilities dependent only on decisive structures does not guarantee split-proofness. Since the calculations are tedious, they are skipped here.

Example 2. Consider any 3-party system. Suppose the probabilities of recognition are dependent solely on the decisive structure of the game and are equal to Shapley values for each of the parties. It can be shown that every non-degenerate case of 3-party systems is vulnerable to splits. Basically, a party that is pivotal, but has surplus majority, can divide itself into 2 parties each of them being pivotal in the new configuration.

C. Split-proof rule. In this section I will present a recognition rule that is completely split-proof, anonymous and weakly increasing. First note the following 2 facts:

1. "Worst case scenario": If probability of recognition $p_{p}$ for a party $p$ is non-zero, it has strictly positive continuation value $V_{p}$. Since in the worst possible case the party has to offer $\delta\left(1-V_{p}\right)$ and additionally might be always offered zero if selected by other parties, the minimal achievable continuation value as the function of recognition probability satisfies

$$
\begin{gathered}
V_{p}^{W}=p_{p}\left(1-\delta\left(1-V_{p}^{W}\right)\right), \\
V_{p}^{W}=\frac{p_{p}-p_{p} \delta}{1-p_{p} \delta} .
\end{gathered}
$$

2. "Best case scenario": If, on the other hand, the party is always offered non-zero payment and does not have to pay anything to get votes of majority if being selected as the proposer, the maximal continuation value (given $p_{p}$ ) satisfies

$$
\begin{gathered}
V_{p}^{B}=p_{p} \cdot 1+\left(1-p_{p}\right) \delta V_{p}^{B}, \\
V_{p}^{B}=\frac{p_{p}}{1-\delta+p_{p} \delta} .
\end{gathered}
$$

Now we can construct the split-proof rule. The rule will assign probabilities of recognition in relation to size of each party. We will construct the following increasing sequence $\left(\pi_{i}\right)_{i=1}^{n}$, where $n$ is the total number of players:

- start with some small $\pi_{1}=\varepsilon$,

- $\pi_{i+1}=\frac{x}{1-\delta+\delta x}$, where $x=\frac{2 \cdot \pi_{i}}{1-\delta+\pi_{i} \delta}$,

- continue until size of the set $n$ is reached. 
For each party system, probability of recognition for a party having $s$ players is equal to $\pi_{s}$ with $\varepsilon$ chosen so that the probabilities sum up to 1 . The way in which the sequence was constructed guarantees that splitting would make the splitting party worse off: sum of continuation values is constant and the construction maintains strong relative differences between parties with different number of players. Any 2 parties with smaller number of players have a lower sum of valuations than any party with even only one more seat.

The rule proposed here is of course very crude and makes probability of recognition for smaller parties extremely low compared to their counterparts with only slight advantage in terms of number of players.

D. General split-proof and merger-proof rule. The rule is based on a very simple principle. As it was shown before, it is not enough to choose recognition probabilities that are proportional to weights to prevent splits and mergers, as what really matters in this calculus are continuation values. However, if we can choose recognition probabilities so that the continuation values are proportional to the weights, we are done! We just apply an inverse function that transformed the recognition probabilities into continuation values and for any vector of weights we get the vector of probabilities that assures stability. Since, given the assumptions we made, the function is onto the appropriate set, the existence is assured. The following is an example of application of such rule:

EXAmple 4. 3 parties, weights (numbers of players) satisfying $1 / 2>s_{1}>s_{2}>s_{3}$.

In this case, any 2 parties can form a majority coalition. Given the weights, the conditions on probabilities of recognition are given by:

$$
\begin{gathered}
p_{1}\left(1-\delta s_{3}\right)+0=s_{1}, \\
p_{2}\left(1-\delta s_{3}\right)+s_{3} \delta s_{2}=s_{2}, \\
p_{3}\left(1-\delta s_{2}\right)+\left(s_{1}+s_{2}\right) \delta s_{3}=s_{3},
\end{gathered}
$$

which gives the following solution after a simple transformation:

$$
p_{1}=\frac{s_{1}}{1-\delta s_{3}}, \quad p_{3}=\frac{s_{3}(1-\delta)}{1-\delta+\delta s_{1}}, \quad p_{2}=1-p_{1}-p_{3} .
$$

Note that the above method is more general: in this case the vector of continuation values is equal to weights of the players, but one might find recognition rules which produce also continuation values corresponding to e.g. Shapley values. The more formal formulation of the result follows.

THEOREM. There exists a unique class of recognition rules which are stable. They are characterized by continuation values which for each party in the initial party structure are equal to the number of players belonging to that party.

The result that if the condition on continuation values is not satisfied then such a recognition rule is not stable is straightforward. Any exception to equity similar to the split-proof rule presented in $\mathrm{C}$ would be vulnerable to mergers, and a similar principle applies the other way round. Consider the following two initial party systems: $s=\left(s_{1}, s_{2}\right.$, $\left.\ldots, s_{p}, s_{p+1}, s_{p+2}, \ldots, s_{k}\right)$, with $k$ parties and $s^{\prime}=\left(s_{1}, s_{2}, \ldots, s_{p}+s_{p+1}, s_{p+2}, \ldots, s_{k}\right)$, with $k-1$ parties. The only way to prevent splits if the initial system is $s^{\prime}$ and mergers if the initial system is $s$ is to have the sum of continuation values for parties $p$ and $p+1$ 
for system $s$ to be equal to the continuation value of the corresponding party with size $s_{p}+s_{p+1}$ for system $s^{\prime}$. As by anonymity labeling does not matter and the same must be true for any pair of parties for any given system of any size $k$ and a corresponding system of size $k+1$ which differ only by the fact that one of its parties split, it follows the continuation values must be equal to weights.

Now we need to prove that there exist recognition probabilities that will result in the continuation values we need for stability. We will use the following lemma:

LEMma. For any vector of continuation values $V=\left(V_{1}, V_{2}, \ldots, V_{p}, \ldots, V_{k}\right)$, there exists a vector of probabilities of recognition $p=\left(p_{1}, p_{2}, \ldots, p_{p}, \ldots, p_{k}\right)$ resulting in payoffs for parties equal to $V$.

The proof will be by construction. For each of the parties we will have the following condition:

$$
p_{p}\left(1-\min _{C \in D_{p}}\left(\sum_{l \in C} \delta V_{l}\right)\right)+q_{p} \delta V_{p}=V_{p} .
$$

However, now the continuation values are known and we need to find relevant probabilities. Order the parties in terms of their required continuation values. As the continuation values are known, it is uniquely determined what value of the cheapest coalition partners needed by each party to gain majority is. It means the value of $\min _{C \in D_{p}}\left(\sum_{l \in C} \delta V_{l}\right)$ is constant. We will denote the value of cheapest partners for party $p$ by $c_{p}$. The values of $q_{p}$ are also already determined unless in some situation two different sets of coalition partners cost the same (e.g. 2 parties have the same continuation values). If this is the case, make any assumption on the probabilities of choosing the respective alternatives and apply it consistently to each equation. Then, as everything else is known, simply calculate the respective values of probabilities:

$$
p_{p}=\frac{V_{p}\left(1-q_{p} \delta\right)}{1-\min _{C \in D_{p}}\left(\sum_{l \in C} \delta V_{l}\right)} .
$$

It is easy to check that the probabilities sum up to 1. By adding all the conditions for each party we will get the following, as the sum of continuation values equals 1 :

$$
\sum_{p \in K} p_{p}-\delta \sum_{p \in K} p_{p} c_{p}+\delta \sum_{p \in K} q_{p} V_{p}=1 .
$$

However, we have

$$
\sum_{p \in K} p_{p} c_{p}=\sum_{p \in K} q_{p} V_{p}
$$

as the left-hand side sum represents all offers made by players to all other players and the right-hand side sum adds all the offers received by all players. Now it follows that

$$
\sum_{p \in K} p_{p}=1 \text {. }
$$

Notice that the probabilities are well-defined no matter how we allocate probabilities $q_{p}$ in those situations in which players are indifferent between 2 or more sets of cheapest coalition partners. That is the reason why in the theorem we talk about a class of recognition rules. However, each recognition rule within such a class corresponds to the same continuation values. 
The lemma is much more general than we need. As it applies to any vector of continuation values it is in particular true for continuation values that are equal to weights of parties (proportion of players belonging to each of them). This ends the proof.

Notice that this rule does not allow for a majority party to be the default winner in each case. Any such recognition rule would be vulnerable to mergers as by forming a majority party it would automatically exclude other parties and make their expected payoffs equal to 0 .

5. Summary. In this paper we evaluated implications of different recognition rules, concentrating on the impact they have on stability of party systems. The common assumption that the probability of recognition is proportional to weights (number of seats in a legislature or number of stocks) is not sufficient for stability, neither is making a rule dependent only on the decisive structures. It can be replaced with a similar principle related to continuation values which provides the only rule ensuring stability. Since such a situation is unlikely, it suggests that treating parties as single players in such a setting constitutes a significant constraint imposed on players' actions.

\section{References}

[1] D. P. Austen-Smith and J. S. Banks, Elections, coalitions, and legislative outcomes, American Political Science Review 82 (1988), 405-22.

[2] J. S. Banks and J. Duggan, A bargaining model of collective choice, American Political Science Review 94 (2000), 73-88.

[3] J. S. Banks and J. Duggan, A bargaining model of policy-making, mimeo, 2001.

[4] D. P. Baron, A spatial bargaining theory of government formation in a parliamentary system, American Political Science Review 85 (1991), 137-64.

[5] D. P. Baron, Government formation and endogenous parties, American Political Science Review 87 (1993), 34-47.

[6] D. P. Baron and J. Ferejohn, Bargaining in legislatures, American Political Science Review 83 (1989), 1181-1206.

[7] D. Diermeier and A. Merlo, An empirical investigation of coalitional bargaining procedures, mimeo, 1999a.

[8] D. Diermeier and A. Merlo, A Structural model of government formation, mimeo, 1999b.

[9] M. O. Jackson and B. Moselle, Coalition and party formation in a legislative voting game, Journal of Economic Theory 103 (2001), 49-87.

[10] A. Merlo and C. Wilson, A stochastic model of sequential bargaining with complete information, Econometrica 63 (1995), 371-399.

[11] A. Merlo and C. Wilson, Efficient delays in a stochastic model of bargaining, Economic Theory 11 (1998), 39-55.

[12] A. Rubinstein, Perfect equilibrium in a bargaining model, Econometrica 50 (1982), 97-109.

[13] L. S. Shapley, A value for n-person game, in: H. W. Kuhn and A. W. Tucker (eds.), Contributions to the Theory of Games, Princeton University Press, 1953. 